\title{
GEOMETRIC PRESENTATIONS OF CLASSICAL KNOT GROUPS
}

\author{
JOHN ERBLAND \\ Department of Mathematics \\ University of Hartford \\ West Hartford, Connecticut 06117 \\ and \\ MAURICIO GUTERRIEZ \\ Mathematics Department \\ Tufts University \\ Medford, Massachusetts 02155 \\ (Recelved September 19, 1989)
}

ABSTRACT. The question addressed by this paper is, how close is the tunnel number of a knot to the minimum number of relators in a presentation of the knot group? A dubious, but useful conjecture, is that these two invariants are equal. (The analogous assertion applied to 3-manifolds is known to be false. [1]) It has been shown recently [2] that not all presentations of a knot group are "geometric". The main result in this paper asserts that the tunnel number is equal to the minimum number of relators among presentations satisfying a somewhat restrictive condition, that is, that such presentations are always geometric.

1. DEFINITIONS AND CONVENTIONS.

Let $K$ be any non-trivial knot in $\mathrm{S}^{3} ; \mathrm{V}=\mathrm{Cl}(\mathrm{N}(\mathrm{K})=$ solid torus containing $\mathrm{K}$; $X=C l\left(s^{3}-v\right)$, the knot exterior.

Let $G=$ the knot group $\pi_{1}\left(s^{3}-K\right)$. If $G$ has the deficiency-1 presentation $\left\langle g_{1}, \ldots, g_{n+1} \mid r_{1}, \ldots, r_{n}\right\rangle$, we will abuse notation and use $g_{1}$ and $r_{j}$ to denote loops representing the corresponding group elements. We restrict consideration to presentations having representative loops $g_{j}$ and $r_{j}$ such that there are disks $D_{j} X$ with $\partial D_{j}=r_{j}$ and $\operatorname{int}\left(D_{j}\right) g_{1}=\emptyset$ for all $1, j$. Let $s=s(K)=$ minimum number of relators in such a presentation. $X_{0}=\operatorname{Cl}\left(N\left(g_{1}\right)\right.$ ), a neighborhood of a "bouquet" of generators, is a handlebody of genus $s+1$.

Let $t=t(K)$ be the tunnel number of $K$, which is the minimum number of arcs that need to be attached to $K$ so that the complement is an open handlebody. Equivalently, $t(K)$ is the minimum number of 1 -handles (tunnels) that need to be removed from the knot exterior to obtain a Heegard decomposition of $s^{3}$. $t(K)$ is also called the "Heegard" genus of $\mathrm{K}[3,2]$. 
2. THEOREM: $s(K)=L(K)$ for any tame knot $K$.

The direction $s(K)<t(K)$ is easy: The fundamental group of the complement of $K$ together with the $t=t(K)$ arcs is free on $t+1$ generators. For each arc, a loop around that arc provides a relation. Such a presentation is called geometric.

Outline of proof, of $t(K)<s(K)$ : For each $r_{j}$, the corresponding disk $D_{j}$ can be moved so that $\partial D_{j} \subseteq \partial x_{0}$, int $\left(D_{j}\right) \subseteq S^{3}-x_{0}$. We bulld up a space $x_{s}$ from $x_{0}$ by stages using 2-handle surgeries along these disks; $x_{j}$ is the result of the jth surgery. $x_{s}$ is the result of the last surgery, with $s^{3}-x_{s}=N^{\prime}(K)$, a solid torus neighborhood of $K$. Thus a Heegard decomposition of $\mathrm{s}^{3}$ can be obtained by drilling tunnels through the disks used in the 2-handle surgeries.

The rest of this paper is concerned with filling in the details.

3. THE CONSTRUCTION.

Let $x_{1}=x_{0} \cup C_{1}\left(N\left(D_{1}\right)\right)$, by 2-handle surgery along $D_{1} ; D_{1}^{\prime}=D_{1}$. For each $j>1$, for each $1<j$, if $N\left(D_{i}^{\prime}\right) \quad D_{j} \neq$ then modify $D_{j}$ to remove intersections. (Fig. la,b). Of course, there may be more intersections, but they must all be simple arcs

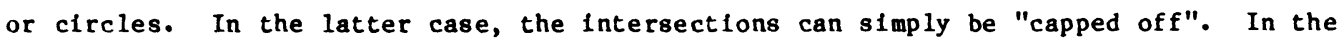
case of multiple arcs, start with the outermost arc of the new disk and continue until all of the intersections have been removed. The modification may result in an increase in the number of disks. If any disk is "trivial", simply "throw it out". We consider a disk to be trivial if the union of the disk with $x_{j-l}$ and previous disks contains an incompressible 2-sphere. The determination of which disks are trivial is not unique: after $D_{j}$ has been modified, if extra disks result, then select one at a time. If you get an incompressible sphere, discard that disk from consideration and move on to the next.

Let $D_{j}$ ' be the union of disks obtained from $D_{j}$ after modification then removal of trivial disks. For $j>1$, let $x_{j}$ be the space obtalned from $x_{j-1}$ by 2-handle surgeries along $D^{\prime}{ }_{j}$. By minimality of $s$, at least $s$ 2-handle surgeries are performed. The boundary of $x_{j}$ differs from that of $x_{j-1}$ either'by a decrease in the genus of one component or by an increase in the number of components. At the same time none of the boundary components can be a 2-sphere. Thus the end result of the construction, $X_{s}$, is bounded by tori only: $\partial X_{s}=T_{0} \cdots T_{k}$. We need to show that $k=0$, where $T_{0}$ bounds a solid torus $N^{\prime}(K)$.

4. Before finishing the proof, we make some essential observations.

(i) $\pi_{1}\left(X_{s}\right) \simeq \pi_{1}(X)=G$, the isomorphism being induced by inclusion, follows from a standard Seifert-Van Kampen argument.

(ii) There is no 2-sphere $S \subseteq X_{s}$ that separates two of these tor1. This follows from the construction itself.

(1ii)For each $i, \pi_{1}\left(T_{1}\right)+\pi_{1}\left(x_{s}\right) 18$ infective. Otherwise there exists an essential loop $\alpha$ in $T_{1}$ and a disk $D$ in $x_{s}$ bounded by $\alpha$. $D \times\{-1,1\}$ together with part of the torus is a sphere that can be moved away from the torus and separates it 
from the other tori, contradicting remark (ii).

(iv) Each corus bounds a solid torus in $\mathrm{s}^{3}$ on one side and hence a (possibly trivial) knot exierior on the other [4]. If $\mathfrak{i}>0$, then the solid side of $T_{1}$ must contain the knot; otherwise, there exists a contractible loop in the exterior of $K$ which is not contractible in $x_{s}$, thus contradicting ( $i$ ).

(v) For $i>1$, let $B_{i}=$ component of $s^{3}-x_{s}$ bounded by $T_{i}$. Let $B_{0}$ be the component of

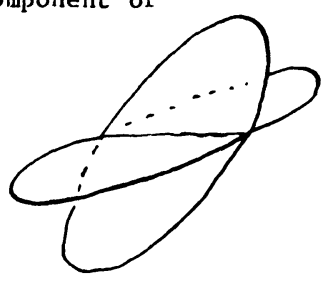

(a)

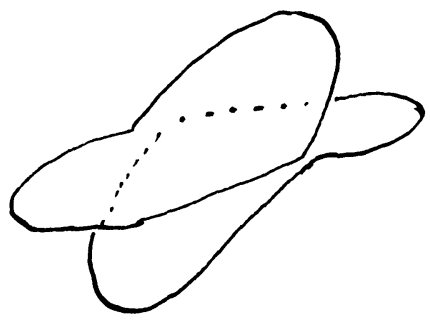

(b)

\section{Figure 1}

$X-X_{s}$ bounded by $T_{0} \partial N(K)$. By (iv) $B_{i}$ is a knot exterior for $i>1$, hence $\pi_{1}\left(T_{1}\right) \rightarrow \pi_{1}\left(B_{1}\right)$ is injective. For related reasons, $\pi_{1}\left(T_{0}\right) \pi_{1}\left(B_{0}\right)$ is injective.

5. We will now proceed to show that $B_{1}=\emptyset$ for $1>0$.

Let $\mathrm{W}_{0}=\mathrm{X}_{\mathrm{s}} \mathrm{B}_{0}, \mathrm{~W}_{\mathrm{j}}=\mathrm{W}_{\mathrm{j}-1} \mathrm{~B}_{\mathrm{j}}$ for $\mathrm{j}>0$. In particular, $\mathrm{W}_{k}=\mathrm{X}$.

For $i>0$, we apply the Seiferi-Van Kampen Theorem to the injectivity of $\pi_{1}\left(T_{1}\right) \rightarrow \pi_{1}\left(X_{s}\right)$ and $\pi_{1}\left(T_{i}\right)+\pi_{1}\left(B_{i}\right)$ to get injectivity of $\pi_{1}\left(B_{1}\right)+\pi_{1}\left(X_{s} B_{i}\right)$ and $\pi_{1}\left(X_{s}\right)+\pi_{1}\left(X_{s} B_{1}\right)$. In particular, $\pi_{1}\left(X_{s}\right) \rightarrow \pi_{1}\left(W_{0}\right)$ is injective. We apply the inductive hypothesis that $\pi_{1}\left(x_{s}\right)+\pi_{1}\left(w_{j-1}\right)$ is injective, together with another application of Seifert-Van Kampen, to get injectivity of $\pi_{1}\left(W_{j-1}\right)+\pi_{1}\left(W_{j}\right)$ and of $\pi_{1}\left(X_{s} \cup B_{j}\right)+\pi_{1}\left(w_{j}\right)$. Since $w_{k}=X$, we have

$$
\pi_{1}\left(X_{s}\right)+\pi_{1}\left(W_{0}\right)+\pi_{1}\left(W_{1}\right)+\ldots+\pi_{1}(X)=G
$$

Since this composition is an isomorphism, all of the component injections must also be isomorphisms. From this and the Seifert-Van Kampen construction, it follows that $\pi_{1}\left(X_{s}\right) \simeq \pi_{1}\left(X_{s} B_{1}\right)$ and hence $\pi_{1}\left(B_{1}\right) \simeq \pi_{1}\left(T_{1}\right)$ for $1>0$, which is impossible for a knot exterior. Thus only $B_{0}$ is non-empty.

6. It remains to verify that $\operatorname{tnt}\left(B_{0} \quad V\right)=N^{\prime}(K)$, just a fatter neighborhood of $K$, thus completing the proof.

The boundary of $B_{0}$ is the union of two tori, one of which $t s \quad \partial N(K)$. The inclusions in both cases must induce isomorphisms of fundamental groups. Since $\pi_{1}\left(B_{0}\right) \simeq \pi_{1}\left(T_{0}\right) \simeq \pi_{1}(\partial N(K)) \simeq Z+z$ is abelian, the Hurewicz homomorphism is an 
isomorphism onto $H_{1}\left(B_{0}\right)$. It follows that the meridional loops $m_{1}$ and $m_{2}$ on the bounding tori are homologous. Let $\alpha$ be a path from the base point of $m_{1}$ to the base point of $m_{2}$. Then $m_{1} \alpha m_{2}^{-1} \alpha^{-1}$ is homogous to 0 and hence homotopically trivial. Thus the meridians bound an annulus and the torus $T_{0}$ is parallel to $\partial N(K)$, which suffices to complete the proof.

\section{REFERENCES}

1. MONTESINOS, J.M. Note on a result of Bolleau-Zleschang, in Low-dimensional Topology and Kleinian Groups, LMS Lecture Notes Ser. 112 (1986), $\overline{241-252 .}$

2. BOIleAU, M., ROST, M., ZIESCHANG, H. On Heegard Decompositions of Torus Knot Exteriors and Related Selfert Fibre Spaces, Math. Ann. 279 (1988), 553-581.

3. CLARK, B. The Heegard genus of manifolds obtained by surgery on links and knots Int. J. Math. \& Math. Sci. 3 (1980), 583-589.

4. ROLFSEN, D. Knots and Links, Publish or Perish Inc. (1976). 


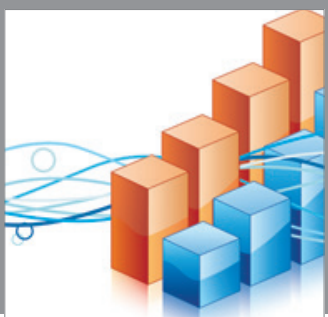

Advances in

Operations Research



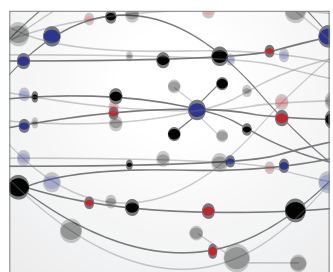

\section{The Scientific} World Journal
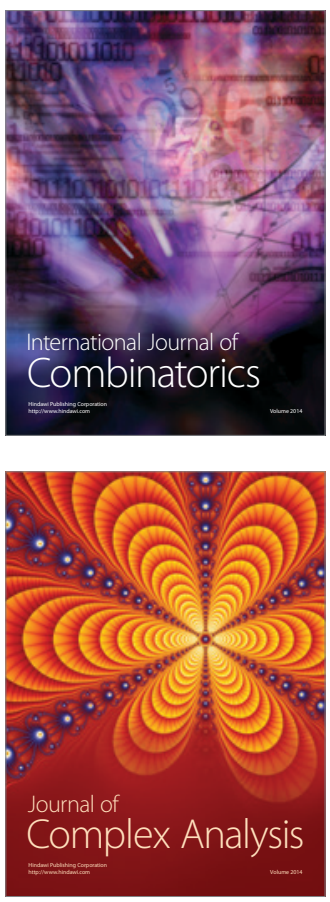

International Journal of

Mathematics and

Mathematical

Sciences
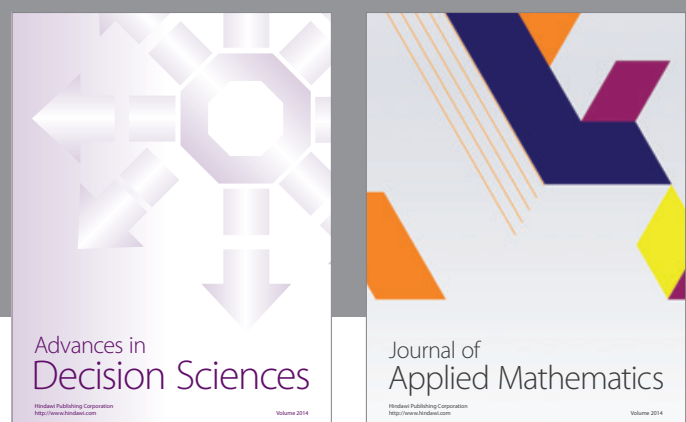

Journal of

Applied Mathematics
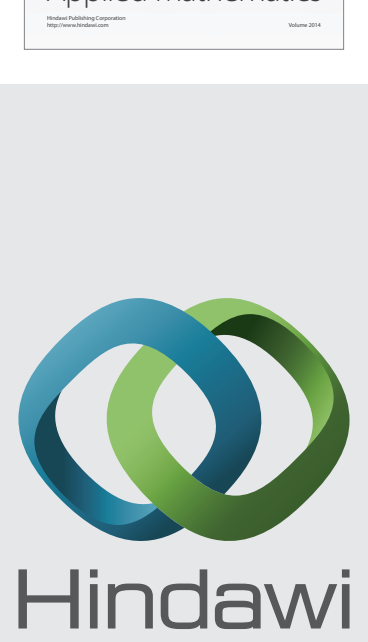

Submit your manuscripts at http://www.hindawi.com
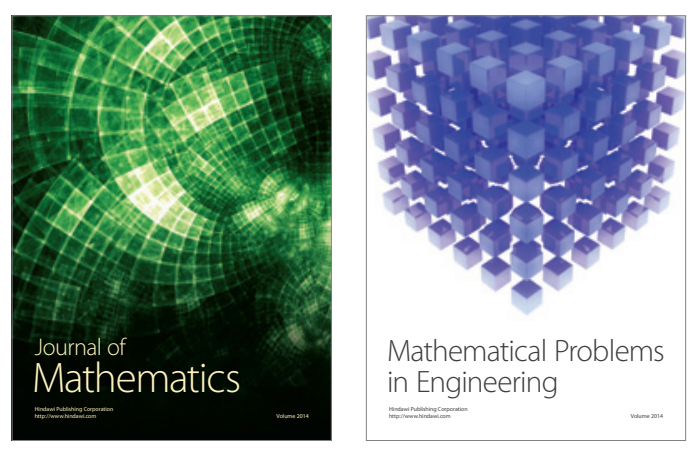

Mathematical Problems in Engineering
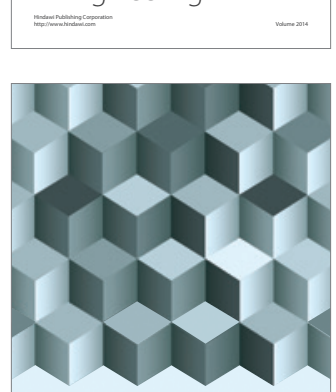

Journal of

Function Spaces
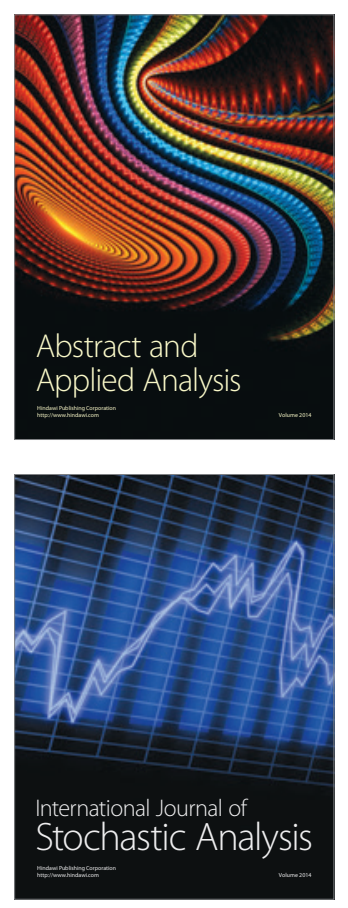

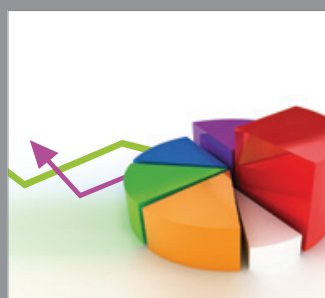

ournal of

Probability and Statistics

Promensencen
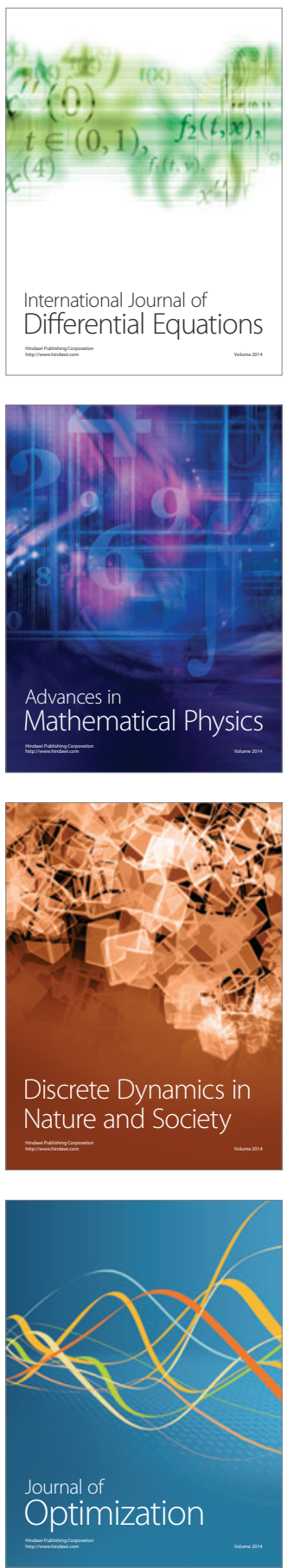